\title{
Upper chromospheric magnetic field of a sunspot penumbra: observations of fine structure
}

\author{
J. Joshi ${ }^{1,2}$, A. Lagg ${ }^{2}$, S. K. Solanki ${ }^{2,3}$, A. Feller², M. Collados ${ }^{4,5}$, D. Orozco Suárez ${ }^{4,5}$, R. Schlichenmaier ${ }^{6}$, M. Franz ${ }^{6}$, \\ H. Balthasar ${ }^{7}$, C. Denker ${ }^{7}$, T. Berkefeld ${ }^{6}$, A. Hofmann ${ }^{7}$, C. Kiess ${ }^{6}$, H. Nicklas ${ }^{8}$, A. Pastor Yabar ${ }^{4,5}$, R. Rezaei ${ }^{4,5}$, \\ D. Schmidt ${ }^{6}$, W. Schmidt ${ }^{6}$, M. Sobotka ${ }^{9}$, D. Soltau ${ }^{6}$, J. Staude ${ }^{7}$, K. G. Strassmeier ${ }^{7}$, \\ R. Volkmer ${ }^{6}$, O. von der Lühe ${ }^{6}$, and T. Waldmann ${ }^{6}$
}

1 Institute for Solar Physics, Department of Astronomy, Stockholm University, AlbaNova University Centre, 10691 Stockholm, Sweden,

e-mail: jayant.joshi@astro.su.se

2 Max-Planck-Institut für Sonnensystemforschung, Justus-von-Liebig-Weg 3, 37077 Göttingen, Germany

3 School of Space Research, Kyung Hee University, Yongin, 446-701 Gyeonggi Do, Republic of Korea

4 Instituto de Astrofísica de Canarias (IAC), Vía Lactéa, 38200 La Laguna, Tenerife, Spain

5 Departamento de Astrofísica, Universidad de La Laguna, 38205 La Laguna, Tenerife, Spain

${ }^{6}$ Kiepenheuer-Institut für Sonnenphysik, Schöneckstr. 6, 79104 Freiburg, Germany

7 Leibniz Institut für Astrophysik Potsdam (AIP), An der Sternwarte 16, 14482 Potsdam, Germany

8 Institut für Astrophysik, Georg-August-Universität Göttingen, Friedrich-Hund-Platz 1, 37077 Göttingen, Germany

9 Astronomical Institute of the Academy of Sciences, Fričova 298, 25165 Ondřejov, Czech Republic

Received 30 June 2016 / Accepted 5 August 2016

\begin{abstract}
Aims. The fine-structure of the magnetic field in a sunspot penumbra in the upper chromosphere is to be explored and compared to that in the photosphere.

Methods. Spectropolarimetric observations with high spatial resolution were recorded with the 1.5-m GREGOR telescope using the GREGOR Infrared Spectrograph (GRIS). The observed spectral domain includes the upper chromospheric He I triplet at $10830 \AA$ and the photospheric Si I $10827.1 \AA$ and Ca I $10833.4 \AA$ spectral lines. The upper chromospheric magnetic field is obtained by inverting the He I triplet assuming a Milne-Eddington-type model atmosphere. A height-dependent inversion was applied to the Si I $10827.1 \AA$ and Ca I $10833.4 \AA$ lines to obtain the photospheric magnetic field.

Results. We find that the inclination of the magnetic field varies in the azimuthal direction in the photosphere and in the upper chromosphere. The chromospheric variations coincide remarkably well with the variations in the inclination of the photospheric field and resemble the well-known spine and interspine structure in the photospheric layers of penumbrae. The typical peak-to-peak variations in the inclination of the magnetic field in the upper chromosphere are found to be $10^{\circ}-15^{\circ}$, which is roughly half the variation in the photosphere. In contrast, the magnetic field strength of the observed penumbra does not vary on small spatial scales in the upper chromosphere.

Conclusions. Thanks to the high spatial resolution of the observations that is possible with the GREGOR telescope at 1.08 microns, we find that the prominent small-scale fluctuations in the magnetic field inclination, which are a salient part of the property of sunspot penumbral photospheres, also persist in the chromosphere, although at somewhat reduced amplitudes. Such a complex magnetic configuration may facilitate penumbral chromospheric dynamic phenomena, such as penumbral micro-jets or transient bright dots.
\end{abstract}

Key words. sunspots - Sun: activity - Sun: chromosphere - Sun: infrared - Sun: magnetic fields - techniques: polarimetric

\section{Introduction}

In photospheric layers, the magnetic field of sunspot penumbrae possesses a highly complex structure that varies in strength and orientation on fine spatial scales. It is organized in nearly radially aligned filaments (at least in the inner penumbra) where strong and weak magnetic fields are alternatingly present in azimuthal direction. Similarly, the magnetic field orientation is organized in vertical (spines) and horizontal (interspine) components (Schmidt et al. 1992; Title et al. 1993; Solanki \& Montavon 1993; Martínez Pillet 2000; Langhans et al. 2005; Scharmer \& Henriques 2012; Tiwari et al. 2013). Relations between various physical parameters of penumbrae, such as the magnetic field strength, field orientation, brightness, and plasma flows
(Evershed effect), have been studied for the past few decades, leading to some controversial results (for detailed reviews on the topic see, e.g., Solanki 2003; Borrero \& Ichimoto 2011; Rempel \& Schlichenmaier 2011). A recent study by Tiwari et al. (2013) seems to settle most of the controversies and presented a uniform picture of penumbral filaments. The authors concluded that the penumbral filaments harbor a magnetic field that is horizontal and comparatively weak, except at its head, where the field is relatively vertical, and at the tail, where the field has opposite polarity. Such filaments are, at least in the inner part of the penumbra, embedded in strong and relatively vertical background magnetic field.

Another feature of the photospheric penumbral magnetic field are opposite-polarity patches (compared to the polarity 
of the umbra) at the tails and edges of penumbral filaments (Scharmer et al. 2013; Ruiz Cobo \& Asensio Ramos 2013; Franz \& Schlichenmaier 2013; Tiwari et al. 2013; van Noort et al. 2013; Joshi 2014; Joshi et al. 2016). These opposite-polarity patches are thought to originate from advection of the penumbral field by overturning convection. Overturning convection in penumbrae has been successfully observed (Joshi et al. 2011; Scharmer et al. 2011; Tiwari et al. 2013; Esteban Pozuelo et al. 2015).

The chromosphere and transition region above the sunspot penumbrae display dynamics on arcsec and sub-arcsec scales, such as penumbral micro-jets (Katsukawa et al. 2007; Jurčák \& Katsukawa 2008; Reardon et al. 2013; Vissers et al. 2015; Tiwari et al. 2016) and transient bright dots (Tian et al. 2014). Katsukawa et al. (2007) suggested reconnection between two magnetic components (spines and inter-spines) as a driver of penumbra jets, whereas Tiwari et al. (2016) argued for reconnection between opposite-polarity patches and fields from spines (cf. Magara 2010). Tian et al. (2014) also speculated that the bright dots that they observed in the penumbra may originate from heating of plasma to transition region temperature by reconnection of magnetic fields from spine and interspine regions. In the literature small-scale transient events in the chromosphere and transition region above sunspot penumbrae are commonly linked to the complex magnetic structure in the photosphere. The question is whether the chromospheric magnetic field of the penumbrae plays a role in these dynamics. This topic has not been investigated because information about the magnetic field in the fine structure of sunspots at chromospheric heights is not as plentiful as information on the magnetic field in the photosphere, especially in terms of spatial resolution. The general structure of sunspot magnetic fields has been studied by Rüedi et al. (1995), Joshi (2014), Schad et al. (2015) and Joshi et al. (in prep.) by using observation in the He I triplet at $10830 \AA$.

In this paper, we present the first maps with high spatial resolution of the penumbral magnetic field in the upper chromosphere obtained from the He I triplet at $10830 \AA$ observed with the 1.5-m GREGOR telescope.

\section{Observations}

We observed a part of a sunspot penumbra in the active region NOAA 12096 on 25 June 2014 with the 1.5-m GREGOR telescope (Schmidt et al. 2012). Spectropolarimetric observations were recorded with the GREGOR Infrared Spectrograph (GRIS, Collados et al. 2012) combined with the Tenerife Infrared Polarimeter-2 (TIP-2, Collados et al. 2007). The GREGOR telescope is equipped with the GREGOR adaptive optics system (GAOS, Berkefeld et al. 2012, 2010), which worked during the observations under good seeing conditions.

We observed a $18 \AA$ wide range centered on $10833 \AA$ with a spectral sampling of $18 \mathrm{~m} \AA$. The observed field of view (FOV) was obtained by a raster scan of the $63^{\prime \prime} .3$ long slit with 60 steps, and a step size of $0^{\prime \prime} 135$. The pixel size along the slit is $00^{\prime \prime} 135$. Full Stokes profiles were recorded with an exposure time of $40 \mathrm{~ms}$, with ten accumulations for every Stokes parameter. The heliocentric coordinates of the centers of the observed FOV were $\left(7^{\circ} \mathrm{N}, 38^{\circ} \mathrm{E}\right)$, so that the cosine of the heliocentric angle is $\mu=0.78$.

Standard data reduction steps have been applied to the raw data (see Collados et al., in prep.), which encompass procedures such as corrections for dark current and flat-field, removal of geometrical distortions and fringes, and polarimetric calibration and cross-talk removal. The continuum level was corrected using a Mcath-Pierce Fourier transform Spectrometer (FTS) spectrum (Livingston \& Wallace 1991; Wallace et al. 1993). The spectral resolution of the recorded spectra is estimated in a similar way as in Borrero et al. (2016) by least-squares fitting the FTS spectrum convolved with a Gaussian spectral point-spread-function (PSF) to the averaged quiet-Sun spectrum (recorded in flat-field mode on the disk center). The best fit between the FTS and averaged quiet-Sun spectrum is found for a PSF with $F W H M=120 \mathrm{m \AA}$ and spectral stray light of $14 \%$. This value of FWHM corresponds to a spectral resolution of $\lambda / \Delta \lambda \simeq 90000$, which is similar to that found by Borrero et al. (2016) for the GRIS/GREGOR observations recorded at $1.56 \mu \mathrm{m}$ (also see Franz et al. 2016).

We estimated the spatial resolution of the observations to be $\sim 0$ '.35 from the spatial power spectrum. For comparison, the diffraction-limited resolution of GREGOR at $10832 \AA$ is $\sim 0^{\prime \prime} .18$, and the best resolution that GRIS can achieve at this wavelength is $\sim 0 \prime 27$ (twice the pixel size). To improve the signal-to-noise ratio of the Stokes parameters, we binned data by two pixels both in the slit direction and in the scanning direction (effective spatial resolution $\sim 0^{\prime \prime} .54$ ) and three pixels in the direction of spectral dispersion. This implies reducing the spatial resolution somewhat, but greatly enhances the signal-to-noise ratio of the data, allowing for much more reliable maps of the magnetic vector in the chromosphere. Figure 1 presents the observed continuum intensity, $I_{c}$, at $10833 \AA$ over the observed FOV (panel a) and the line depression of the blended red components of the He I triplet at $10830 \AA,\left(I_{\mathrm{c}}-I_{\mathrm{He}}\right) / I_{\mathrm{c}}$ (panel e). Stokes $Q, U$, and $V$ maps for the Si I $10827.1 \AA$ line are shown in panels b-d and those for the He I triplet are displayed in panels $\mathrm{f}-\mathrm{h}$.

\section{Inversions}

\subsection{He I $10830 \AA$ triplet}

The He I triplet at $10830 \AA$ originates from the transition between the lower $1 \mathrm{~s} 2 \mathrm{~s}{ }^{3} \mathrm{~S}_{1}$ and the upper $1 \mathrm{~s} 2 \mathrm{p}{ }^{3} \mathrm{P}_{0,1,2}$ energy levels, generating three spectral lines: a blue component at $10829.09 \AA$ and two blended red components at $10830.30 \AA$. The He I triplet is strongly influenced by EUV irradiation from the corona and transition region, capable of ionizing neutral helium atoms, which then, by recombination processes, populate the lower level of the transition (Avrett et al. 1994; Andretta \& Jones 1997; Centeno et al. 2008; Leenaarts et al. 2016).

The He I triplet is thought to be formed near the top of the chromosphere because the UV radiation does not penetrate deeply into the chromosphere, populating the ground state of the He I triplet only in the upper layers. We inverted the He I triplet to obtain the upper chromospheric magnetic field and the Doppler velocities with the HeLIx ${ }^{+}$(Lagg et al. 2004, 2009) inversion code assuming a Milne-Eddington-type model atmosphere, which has proved to be a reasonable approximation for the He I triplet. We used eight free parameters in the model atmosphere to fit the observed Stokes profiles in the He I triplet: magnetic field strength, inclination and azimuth of the magnetic field vector, the Doppler velocity, the Doppler broadening, the damping constant, gradient of the source function, and the ratio between line center and the continuum opacity. The Si I $10827.1 \AA$ line blends with the blue component of the He I triplet. To take this blend into account, we also fit the Si I line together with the He I triplet (for more details see Joshi et al. 2016). 


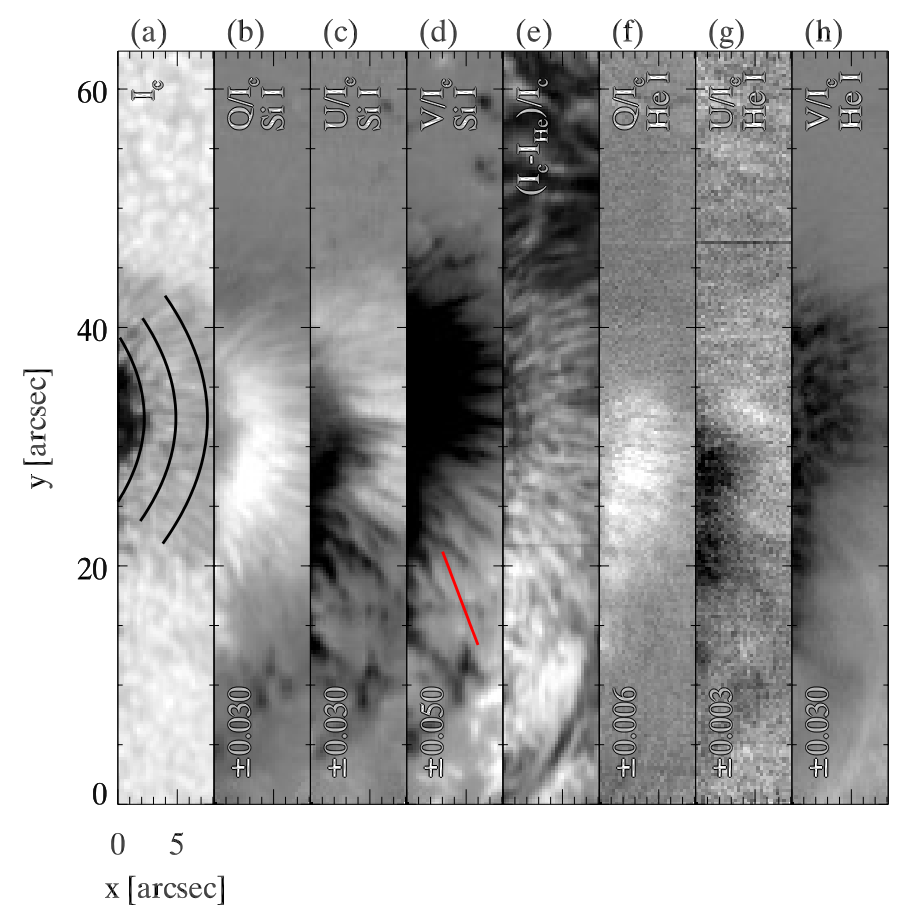

Fig. 1. Maps of Stokes parameters for the Si I $10827.1 \AA$ line and the $\mathrm{He}$ I triplet at $10830 \AA$. Panel a) shows the continuum intensity, $I_{\mathrm{c}}$, at $10833 \AA$. Panels b)-d) corresponds to Stokes $Q / I_{\mathrm{c}}, U / I_{\mathrm{c}}$, and $V / I_{\mathrm{c}}$ maps of the $\mathrm{Si} \mathrm{I}$ line, respectively. The line depression of the blended red components of the He I triplet, $\left(I_{\mathrm{c}}-I_{\mathrm{He}}\right) / I_{\mathrm{c}}$, is displayed in panel e). Stokes $Q / I_{\mathrm{c}}, U / I_{\mathrm{c}}$, and $V / I_{\mathrm{c}}$ maps for the He I triplet are presented in panels $\mathbf{e})-\mathbf{g})$, respectively. The lower and upper limits of the Stokes $Q / I_{\mathrm{c}}, U / I_{\mathrm{c}}$, and $V / I_{\mathrm{c}}$ maps are indicated in the lower part of the respective image. The Stokes maps presented here are an average over a range $0.27 \AA$ wide centered on $\lambda-\lambda_{0}=-3.10 \AA$ and $\lambda-\lambda_{0}=-0.35 \AA$ for the Si I line and He I triplet, respectively, with $\lambda_{0}=10830 \AA$. The Stokes $V / I_{\mathrm{c}}$ profiles along the red line marked in panel d) are plotted in Fig. 2. The lower part of the line is directed toward the closest part of the solar limb. Three arcs in panel a) show the locations of various parameters that are depicted in Fig. 6.

\subsection{Sil $10827.1 \AA$ and $\mathrm{Ca} / 10833.4 \AA$ lines}

To retrieve the height-dependent photospheric atmosphere, simultaneous inversions of the Si I 10827.1 A and Ca I $10833.4 \AA$ lines were performed to satisfy the radiative transfer equation (RTE) under the assumption of local thermodynamic equilibrium (LTE). We used the SPINOR inversion code (Frutiger et al. 2000), which is based on the STOPRO routines (Solanki 1987). We used the same set of line parameters as were used by Joshi et al. (2016).

The model atmosphere used to fit the observed Stokes profiles of the Si I and Ca I lines consists of the temperature, the Doppler velocity, the magnetic field strength, inclination, and azimuth as free parameters at three nodes, $\log \tau_{1083}=$ $0.0,-0.7$, and -2.3 . The micro- and macro-turbulence were assumed to be constant with height. Here $\tau_{1083}$ denotes the optical depth at $1083 \mathrm{~nm}$. The node positions were chosen based on the analysis of the magnetic field response function as carried out by Joshi et al. (2016, also see Joshi 2014) for the lines under consideration.

We are aware that the Si I $10827.1 \AA$ line core forms under non-local thermodynamic equilibrium (NLTE) conditions (see Bard \& Carlsson 2008). Kuckein et al. (2012) have demonstrated that the temperature is the most affected atmospheric parameter when NLTE is not taken into account for the synthesis of this line. The effect on the magnetic field and Doppler velocities is negligible. In this paper, we only discuss the magnetic field and Doppler velocities, justifying the assumption of LTE.

The spectral PSF (plus spectral stray light, 14\%) of the observations is self-consistently included in the SPINOR and $\mathrm{HeLIx}^{+}$inversions. We did not include the spatial PSF of GREGOR and also ignored spatial stray light in the inversions. This washes out contrasts, but should not affect our results qualitatively.

\section{Formation height of the He I triplet}

Stokes $V$ profiles of sunspots observed away from the disk center in a photospheric and a chromospheric spectral line provide a good opportunity to estimate the difference in formation heights of the lines. Line-of-sight (LOS) magnetograms of sunspots observed away from disk center show an apparent neutral line (reversal of polarity) typically located on the limb side of their penumbra. The exact position of the neutral line with respect to the center of the sunspot depends on the view angle and the orientation of the penumbral field. In the typical magnetic field configuration of a sunspot with field lines expanding with height, the Stokes $V$ profiles along the line-of-symmetry (the line joining the disk center and sunspot center) change sign at the location where the magnetic field is perpendicular to the LOS. The He I triplet forming higher in the atmosphere has its neutral line shifted toward the limb compared to that for the lower forming Si I $10827.1 \AA$ line. Knowing the separation between the neutral lines, $\Delta r$, and the viewing angle, $\theta$, we can obtain a rough estimate of the difference in the formation heights, $\Delta h$, using the following formula,

$\Delta h=\frac{\Delta r}{\tan \theta}$,

under the assumption that the polarity inversion line lies at about the same location when projected on the solar surface at both heights. This is the case if the magnetic field (after spatially averaging away the fine structure) at both heights has roughly the same inclination to the vertical (see discussion below).

Figure 2 shows Stokes $V$ profiles both for the Si I $10827.1 \AA$ line and the He I triplet along the line-of-symmetry crossing the neutral line. The bottom and top profiles are located toward the sunspot center and limb, respectively. Profiles colored in blue show the location of the two nearest Stokes $V$ profiles with opposite sign, while profiles in red identify the location of the polarity inversion lines. It is evident that the polarity inversion line for the He I triplet appears closer to the limb than that for the Si I $10827.1 \AA$ line. The separation between the neutral lines is $\sim 1^{\prime \prime} 62$, which is equal to $\sim 1530 \mathrm{~km}$ after correcting for the foreshortening. Therefore, according to Eq. (1), the difference in formation height should be around $1900 \mathrm{~km}$, but Eq. (1) is valid only when the magnetic field orientation with respect to the LOS is the same at both heights. The inclination of the magnetic field obtained from the He I triplet, however, is on average $12^{\circ}$ more vertical than the inclination in the photosphere (see Joshi 2014; Joshi et al., in prep., and Sect. 5 of this article). After including (adding to $\theta$ ) this difference of the inclination angle into the Eq. (1), the difference between the formation height of the He I triplet and Si I line is reduced to around $1250 \mathrm{~km}$. We note that the formation height derived here is specific to the sunspot penumbra and may differ significantly in other solar features.

The Si I $10827.1 \AA$ line has multilobed Stokes $V$ profiles at and close to the periphery of the sunspot $\left(r=44^{\prime \prime} 5-5^{\prime \prime} .5\right)$, similar 
A\&A 596, A8 (2016)

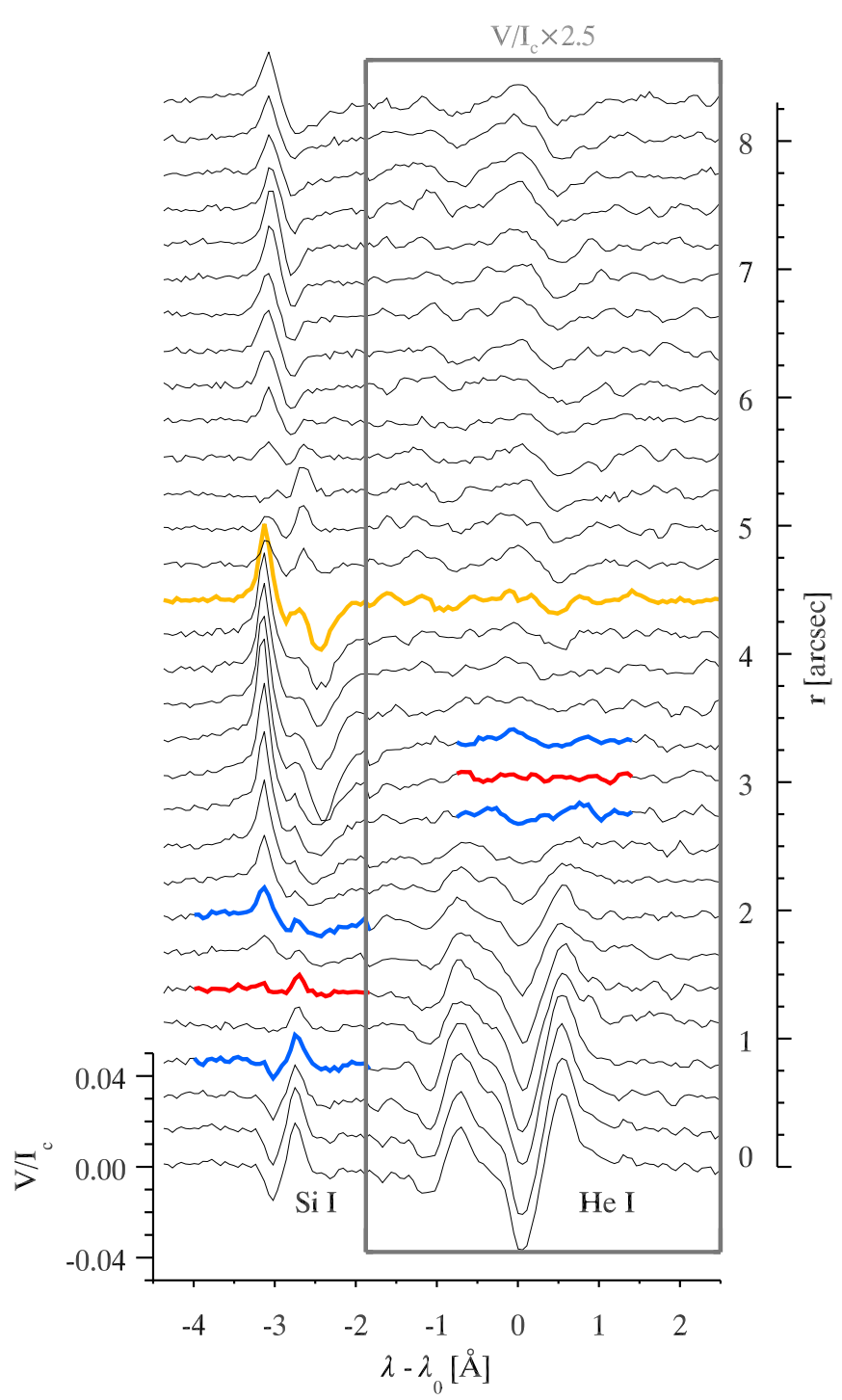

Fig. 2. Stokes $V$ profiles over a spectral range covering the Si I 10827.1 A line and He I triplet at $10830 \AA$ A. Positions of the plotted profiles are indicated by a red line in Fig. 1d, the lowermost profile belongs to the pixel closest within the sunspot to the sunspot center. Since the Stokes $V$ signal in the He I triplet is weaker than the Si I line, it is magnified by a factor of 2.5 (see box). The right side of the axis in the plot gives the distance, $r$, in seconds of arc from the lowermost profile. Profiles plotted in red indicate the position of the polarity inversion line and those colored in blue indicate the nearest profiles with clearly visible opposite polarity. A yellow profile at $r=4$ ".5 marks the transition from the penumbral region to the quiet Sun.

to those close to the polarity inversion line $\left(r=1^{\prime \prime} \cdot 5\right)$. The multilobed Stokes $V$ profiles at the periphery of the sunspot are most probably due to unresolved mixed polarities or a steep gradient in the inclination of the magnetic field with height just outside the sunspot, however. The He I triplet displays normal Stokes $V$ profiles at these locations, indicating that any disturbance of the field is restricted to layers below its formation height.

\section{Results}

Maps of the magnetic field vector and LOS velocity obtained for the photosphere and upper chromosphere are displayed in Fig. 3. The first three maps in each panel correspond to parameters at $\log \tau=0.0, \log \tau=-0.7$ and $\log \tau=-2.3$ obtained by
SPINOR inversions of the Si I $10827.1 \AA$ and Ca I $10833.4 \AA$ lines. The fourth map in each panel shows the parameters returned by $\mathrm{HeLIx}^{+}$inversions of the He I $10830 \AA$ triplet. The magnetic field vectors are transformed from the LOS frame of reference to the solar frame of reference using a transformation matrix provided by Wilkinson et al. (1989). Before the transformation, the $180^{\circ}$ ambiguity in the azimuth direction of the magnetic field was resolved using the acute angle method (Sakurai et al. 1985; Cuperman et al. 1992). The LOS velocities were calibrated by setting the average umbral velocity at $\log \tau=0$ to zero.

All three nodes in the photosphere show the same variation in the magnetic field strength in the azimuthal direction. These alternating weak and strong field structures are aligned nearly in the radial direction. In a similar way, the inclination of the magnetic field is structured, where the relatively horizontal and vertical fields are organized in the same filamentary structure. The upper chromosphere magnetic field strength does not display any variation in azimuthal direction, but the inclination of the field does exhibit such a variation in the azimuthal direction that is qualitatively similar to the variation in the photosphere. The LOS velocities in the photosphere show clear patterns of the radially outward directed Evershed flow, with decreasing velocities from $\log \tau=0.0$ to $\log \tau=-0.7$, and velocities close to zero at $\log \tau=-2.3$. In the upper chromosphere, the LOS velocities have the opposite sign to that in the photosphere, displaying the inverse Evershed flow.

A zoom of the upper chromospheric magnetic field and LOS-velocity maps corresponding to the location indicated by a box in Fig. 3b is presented in Fig. 4. The filamentary structure is apparent in the inclination, but not in the magnetic field strength. Contours of the inclination are overlaid on the maps of $I_{\mathrm{c}},\left(I_{\mathrm{c}}-I_{\mathrm{He}}\right) / I_{\mathrm{c}}, B, \phi$ and $v_{\mathrm{LOS}}$ in panels a-d and $\mathrm{f}$ of Fig. 4, respectively. These contours suggest that the small-scale structures in the inclination map do not have a one-to-one correspondence with any of these parameters.

To check the reliability of the small-scale filamentary structure found in the inclination at upper chromospheric heights from the inversions of the He I $10830 \AA$ triplet, it is important to evaluate the quality of the fits to the observed Stokes profiles. Figure $4 \mathrm{~g}$ depicts the variation in the inclination of the magnetic field along a line plotted in panel d of Fig. 4. To demonstrate the quality of the fits, we chose multiple locations (marked as A-E in Fig. 4g) along this line where the relatively horizontal and vertical field lines appear close to each other and present the corresponding observed and synthesized Stokes profiles in Fig. 5. The match between the observed and synthesized Stokes profiles is exceptionally good for all locations. The variation in the amplitudes of the Stokes profiles also supports the variation in the inclination obtained through the inversions. For example, locations-A and -B have similar amplitudes in Stokes $Q / U$, but the Stokes $V$ amplitude at location-B is larger than that at location-A, suggesting a more vertical field at location-B, similar to the results of the inversions.

For a qualitative comparison between the photospheric and upper chromospheric magnetic field and the LOS velocity, we depict the parameters in Fig. 6 along three arcs (see Fig. 1a) located at the umbra-penumbra boundary, the middle and outer penumbra, respectively. Owing to the viewing angle (away from disk center) and the difference in the formation height $(1250 \mathrm{~km})$ between the Si I line and the He I triplet, parameter maps in the upper chromosphere are shifted by projection toward the limb compared to those in the photosphere. The profiles of the 

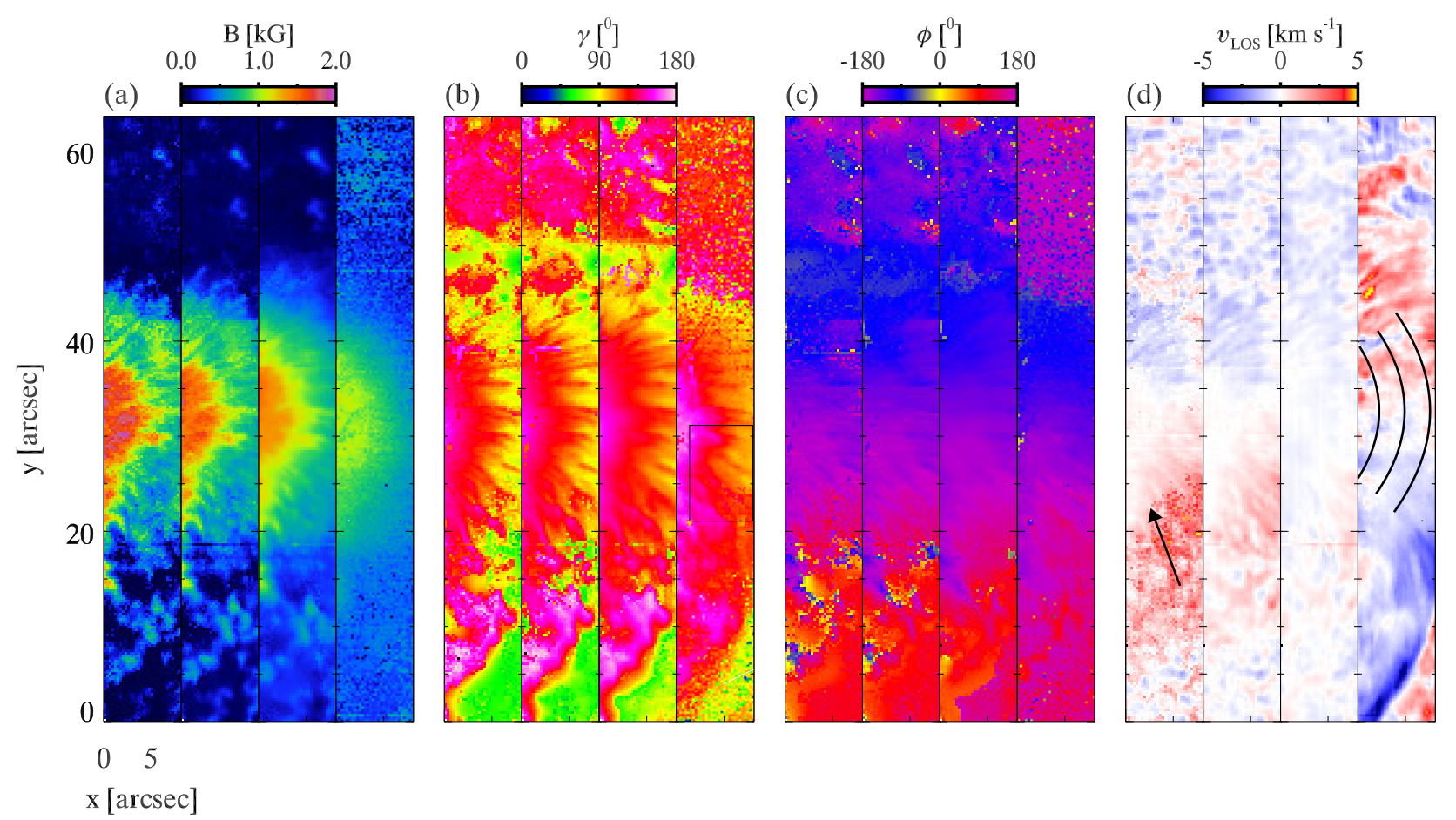

Fig. 3. Maps of the magnetic field vector (from left to right: magnetic field strength, $B$, inclination, $\gamma$, azimuth, $\phi$ ) and LOS-velocity, $v_{\mathrm{LOS}}$, in the photosphere and upper chromosphere. The first three maps in every panel correspond to $\log \tau=0.0, \log \tau=-0.7$ and $\log \tau=-2.3$ (SPINOR inversion of the Si I and Ca I lines), and the fourth map refers to the upper chromosphere (HeLIx ${ }^{+}$inversion of the He I triplet). The box in the fourth map of panel b) represents the location for which details of various parameters are displayed in Fig. 4. Three arcs in panel d) show the locations of various parameters that are depicted in Figs. 6 and 7. An arrow in panel d) indicates the disk center direction.

atmospheric parameters plotted in Fig. 6 are corrected for this shift. The inclination of the magnetic field along the arc at the umbra-penumbra boundary does not vary much in either the upper chromosphere or photosphere. In the middle and outer penumbra, a variation in the inclination angle that is due to spines (vertical field) and interspines (horizontal field) is seen at all plotted photospheric heights. These variations in the photospheric inclination coincide with the variation in the upper chromosphere. The peak-to-peak variation in the inclination decreases from $20^{\circ}-25^{\circ}$ at $\log \tau=0.0$ to $\log \tau=-2.3$ and reaches the lowest value of $10^{\circ}-15^{\circ}$ in the upper chromosphere. At a few locations in the outermost arc, the magnetic field has an inclination lower than $90^{\circ}$ at $\log \tau=0.0$, which means that these locations are of opposite polarity compared to the umbra. Overall, the magnetic field becomes more vertical with height in the photosphere, and in the upper chromosphere it becomes even more vertical by an average angle of $12^{\circ}$ compared to that at $\log \tau=0.0$.

The magnetic field strength varies as a result of spines and interspines at $\log \tau=0.0$ along all the arcs in the penumbra. These variations in the field strength decrease at $\log \tau=-0.7$ and $\log \tau=-2.3$ and completely vanish at the upper chromosphere. Some parts (mostly at interspines and at their edges) of the arcs in the middle and outer penumbra have a higher magnetic field strength at $\log \tau=-0.7$ and $\log \tau=-2.3$ than at $\log \tau=0.0$.

The gas in the arc at the umbra-penumbra boundary is nearly at rest along the LOS at all the photospheric heights, while in the upper chromosphere, the velocity fluctuate strongly, with multiple locations of upflows and downflows (also see Fig. 3). The middle and outer arc indicate small-scale variations in the inverse Evershed flow at chromospheric height. To investigate the relation between these small-scale variations and the inclination angle of the magnetic field, if any, we plot the two quantities along the middle and outer arc in Fig. 7. Both panels in Fig. 7 suggest that the inverse Evershed flow and the inclination vary on similar spatial scales. There are some hints that the stronger flows corresponds to the more horizontal magnetic field and the more vertical field has weaker flows (see for example the first, third to seventh, and eighth dotted vertical line in the left panel and the fifth to ninth line in the panel on the right in Fig. 7). However, this relation between the inclination of the field and the inverse Evershed flow is weak and is not present at all locations.

\section{Summary and discussions}

We have presented the magnetic field structure of a sunspot penumbra in the upper chromosphere at high spatial resolution and have compared it with that in photospheric layers. In the photospheric layers the well-known spine and interspine structure of the penumbrae in the inclination of the magnetic field are clearly visible in our data (Lites et al. 1993; Title et al. 1993; Solanki \& Montavon 1993; Martínez Pillet 2000; Langhans et al. 2005; Scharmer et al. 2013; Tiwari et al. 2013). Our analysis revealed that the spine and interspine structure is more prominent at $\log \tau=0$ than at $\log \tau=-0.7,-2.3$, in agreement with results of Borrero et al. (2008), Scharmer et al. (2013), and Tiwari et al. (2013). The striking result obtained here is that the magnetic field inclination in the upper chromosphere varies. These variations are most prominent along a cut in the azimuthal direction around the sunspot, and they resemble the spine and interspine structure in the photosphere. Our analysis reveals that these variations in the upper chromosphere coincide remarkably well with those in the photosphere, with typical peak-to-peak variations in the inclination of the field of $10^{\circ}-15^{\circ}$, compared to $20^{\circ}-25^{\circ}$ at $\log \tau=0$. 


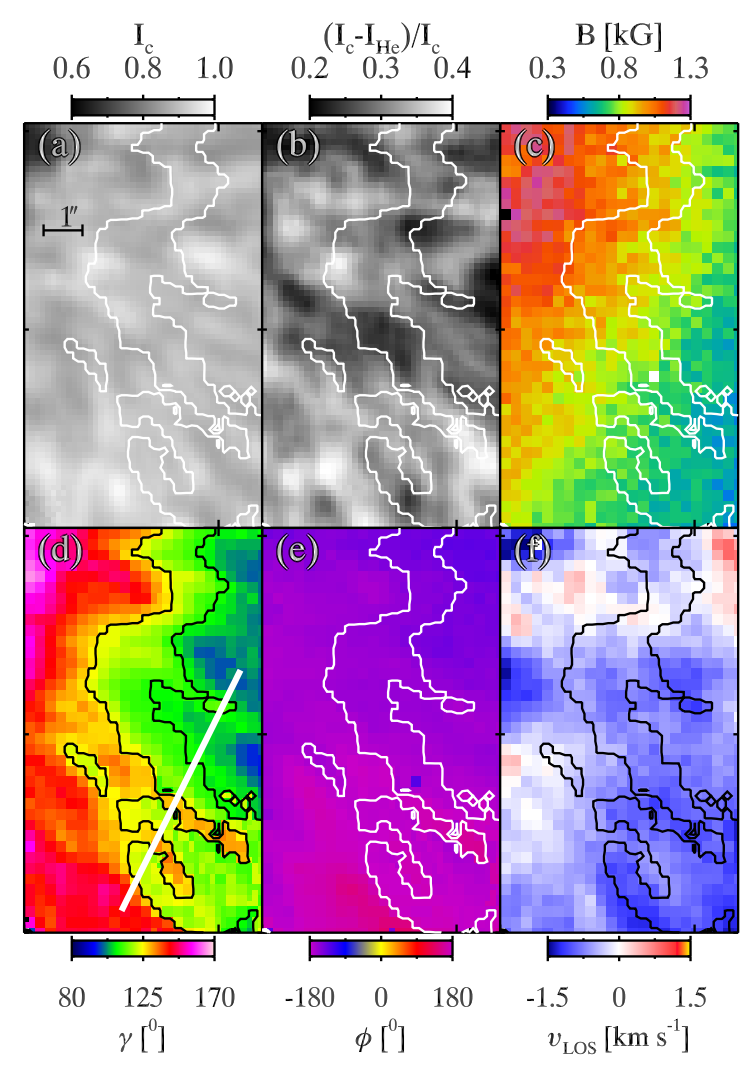

(g)

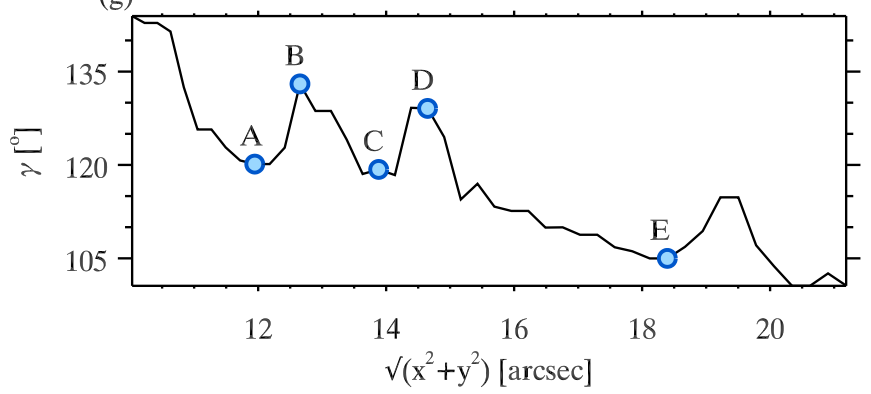

Fig. 4. Details of penumbral fine structure in the upper chromosphere. a) $I_{\mathrm{c}}$, b) $\left(I_{\mathrm{c}}-I_{\mathrm{He}}\right) / I_{\mathrm{c}}$, c) $B$, d) $\gamma$, e) $\phi$, and f) $v_{\mathrm{LOS}}$. The location of the maps is indicated by a box in the fourth map of Fig. 3b. Contours in panels a)-f) depict $\gamma=110^{\circ}$ and $125^{\circ}$. Panel g) depicts variations in $\gamma$ along the white line marked in panel d). Blue circles identified as A-E in panel g) represent locations of observed and synthesized Stokes profiles of the He I triplet plotted in Fig. 5.

A radially aligned filamentary structure in the magnetic field strength is also visible in our results at all photospheric layers with decreasing variation with height. The upper chromospheric map of magnetic field strength does not show any significant variation in the lateral direction, but only a monotonic decrease from the inner to outer penumbra (also see Joshi 2014; Schad et al. 2015). The absence of fine structure in the magnetic field strength map of the penumbra at the upper chromosphere is probably due to the dominance of magnetic pressure over gas pressure at this height and the absence of significant turbulence, contrary to the turbulent photosphere with high plasma- $\beta$ (ratio of plasma pressure to magnetic pressure), where the magnetic field becomes structured on fine spatial scales. The spine and interspine structure found in the magnetic field inclination maps in the upper chromosphere suggest that the orientation of field lines at the photospheric heights is preserved by some extent up

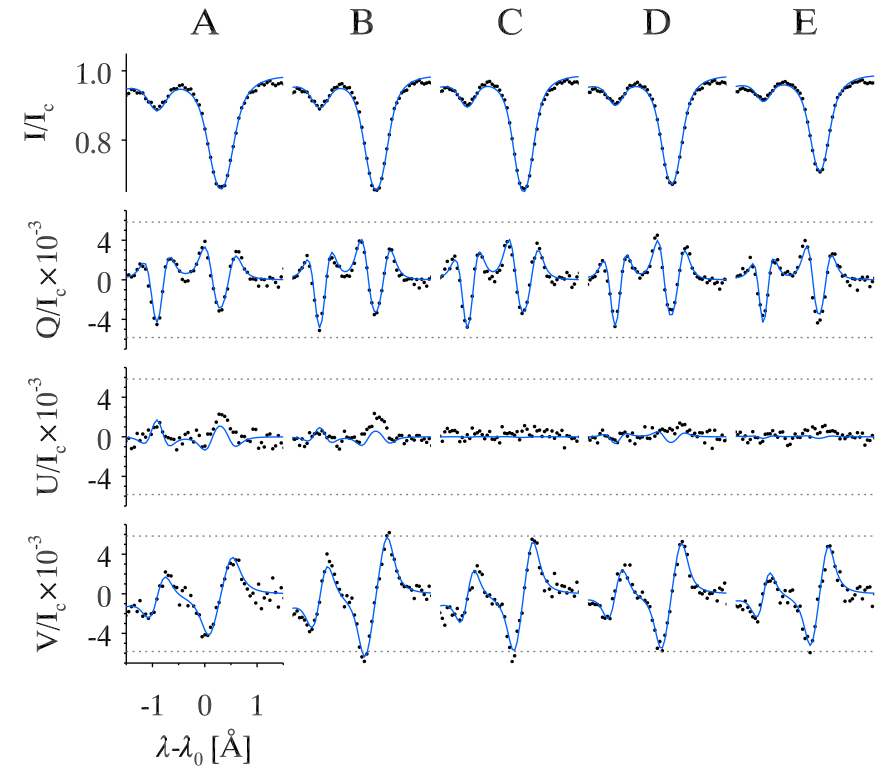

Fig. 5. Observed (black dots) and synthesized (blue curves) Stokes profiles. The computed blue profiles were obtained by the inversions of the He I triplet. Positions of Cols. A-E are marked in Fig. 4g.

to $\sim 1250 \mathrm{~km}$ above the formation height of the Si I $10827.1 \AA$ line.

The observed variations in the magnetic field inclination in the lateral direction might be underestimated at the upper chromospheric height because of the limited resolution of our data. This speculation arises from the fact that our results do not show opposite-polarity (compared to magnetic polarity in the umbra) lanes as observed by Scharmer et al. (2013), Ruiz Cobo \& Asensio Ramos (2013) and Tiwari et al. (2013) at the edges of penumbral filaments in the photosphere. This is because our observations have a lower resolution than the observations analyzed in the above studies. In addition, overlooking the spatial PSF and stray light may contribute to underestimating in the variations in inclination of the magnetic field. However, we do observe some opposite-polarity patches at the tails of penumbral filaments at $\log \tau=0$, similar to those observed by Ruiz Cobo \& Asensio Ramos (2013), Franz \& Schlichenmaier (2013), Scharmer et al. (2013), and Tiwari et al. (2013). Finally, our study also confirms the localized presence of a negative vertical gradient of the magnetic field strength in the photospheric layers of the penumbra, that is, the magnetic field strength decreases with depth as reported by Tiwari et al. (2015) and Joshi et al. (2016). The latter authors interpreted the observed negative vertical gradient as a result of the strong magnetic field from spines closing above the weaker field interspines and also as a result of unresolved opposite-polarity patches in the deep photosphere.

The magnetic field strength, in general, decreases with height in the photosphere and into the upper chromosphere. The average magnetic field strength close to the inner and outer penumbra is about $1.7 \mathrm{kG}$ and $0.8 \mathrm{kG}$ in the photosphere $(\log \tau=0.0)$, respectively, and $1.05 \mathrm{kG}$ and $0.55 \mathrm{kG}$ in the upper chromosphere. If we use the difference between the formation height of the Si I $10827.1 \AA$ line and the He I triplet of $1250 \mathrm{~km}$ that we have determined, then the vertical gradient is $\sim 0.6 \mathrm{G} \mathrm{km}^{-1}$ and $\sim 0.2 \mathrm{G} \mathrm{km}^{-1}$ in the inner and outer parts of the penumbra, respectively. The difference in the formation height of the Si I line and the He I triplet is estimated using the separation between the polarity-inversion lines displayed by these spectral lines. These values of the vertical gradient in the magnetic field strength are 


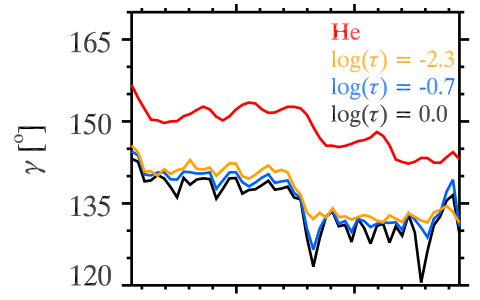

$30 \quad 35$

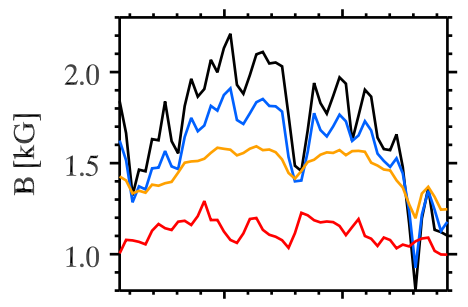

$30 \quad 35$

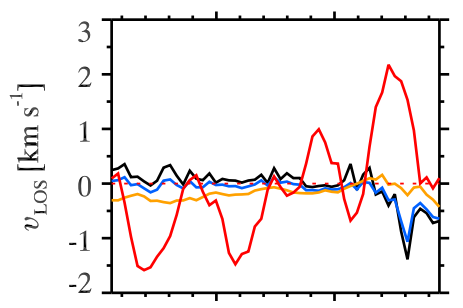

$30 \quad 35$

$\sqrt{ }\left(\mathrm{x}^{2}+\mathrm{y}^{2}\right)[\operatorname{arcsec}]$

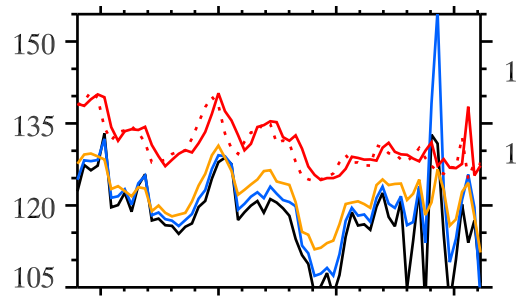

$\begin{array}{llll}25 & 30 & 35 & 40\end{array}$

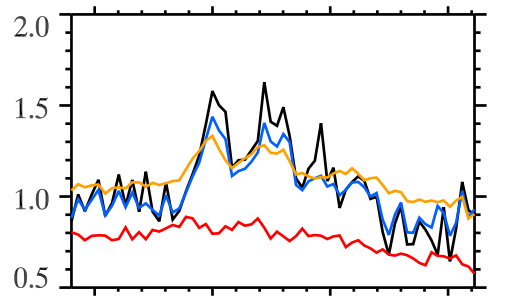

40

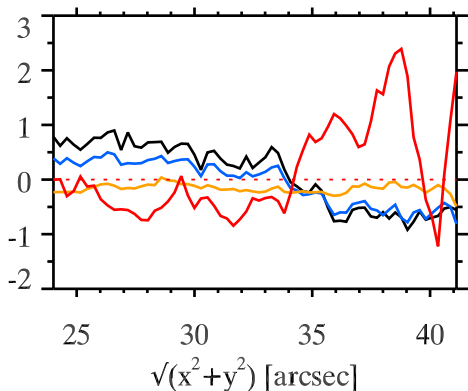

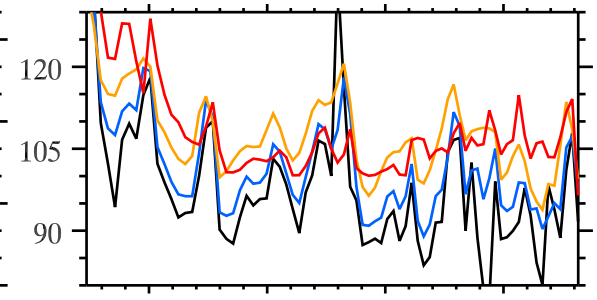

$\begin{array}{llll}25 & 30 & 35 & 40\end{array}$

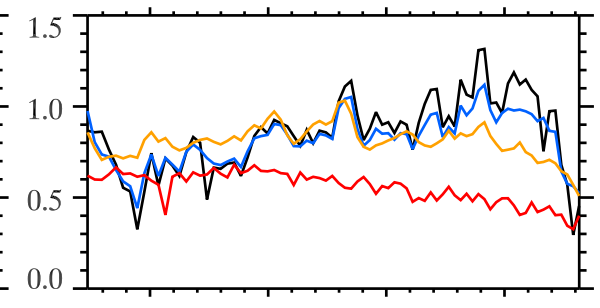

$\begin{array}{llll}25 & 30 & 35 & 40\end{array}$

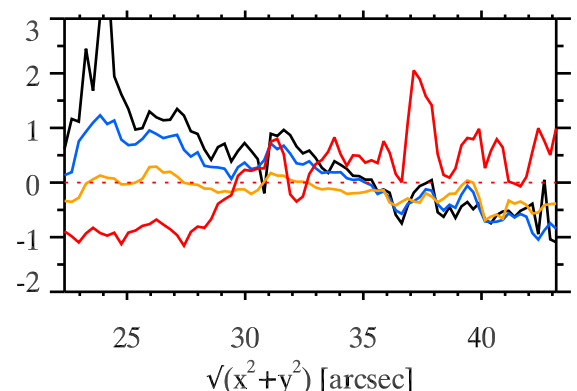

Fig. 6. Profiles of the inclination of magnetic field, $\gamma$ (top row), the magnetic field strength, $B$ (middle row), and the LOS-velocity, $v_{\mathrm{LOS}}$ ( row), along the arcs (see Fig. 1a) at the umbra-penumbra boundary (left column), middle penumbra (middle column), and outer penumbra (right column). Black, blue, yellow, and red curves correspond to $\log \tau=0.0,-0.7,-2.3$, and the upper chromosphere, respectively. All the upper chromospheric parameters are plotted after correcting for the shift that is due to viewing angle and formation height. To illustrate this shift, an uncorrected profile is displayed by the dashed red line in the middle panel of the top row. The left of a panel corresponds to the bottom of the respective arc.
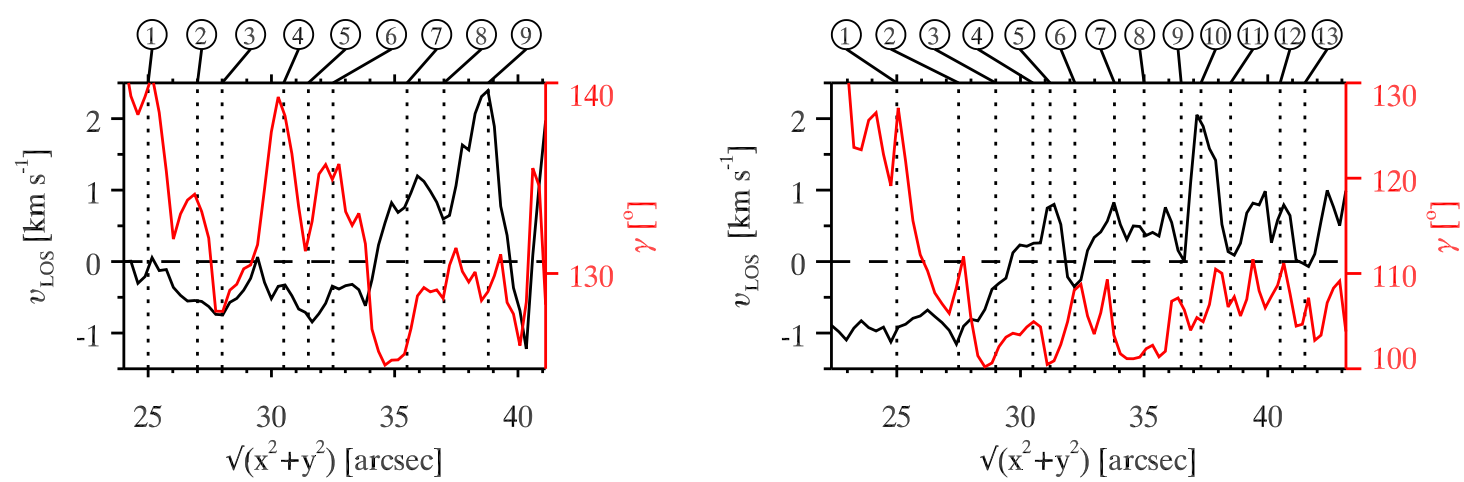

Fig. 7. Variations of the LOS velocity, $v_{\text {LOS }}$ (black curve), and inclination of the magnetic field $\gamma$ (red curve) obtained from the He I triplet, along the middle (left panel) and outer (right panel) arcs shown in Fig. 1a. Numbered vertical lines in both panels represent the locations for which the variations of $v_{\operatorname{LOS}}$ and $\gamma$ are discussed in Sect. 5.

similar to those reported earlier using the same spectral lines (see Rüedi et al. 1995; Joshi 2014; Schad et al. 2015; Joshi et al., in prep.). Our results indicate that in general the magnetic field of the penumbra becomes more vertical with height in the photosphere (also see Borrero \& Ichimoto 2011; Tiwari et al. 2013), and in the upper chromosphere it is more vertical by $\sim 12^{\circ}$ than that in the photosphere $(\log \tau=0.0)$. This difference in the magnetic field inclination between the photosphere and upper chromosphere is similar to that found by Joshi (2014) and Joshi et al. (in prep.).
In summary, we reported the first observations of the fine structure of the penumbral magnetic field of a sunspot in the upper chromosphere, to our knowledge. It displays lateral variation in the magnetic field inclination that resembles the spine and interspine structure in the photosphere. The observed inhomogeneity in the inclination of the chromospheric magnetic field on small scales may play a role in driving small-scale chromospheric and transition region dynamics, such as penumbral micro-jets (Katsukawa et al. 2007; Jurčák \& Katsukawa 2008; Reardon et al. 2013; Vissers et al. 2015; Tiwari et al. 2016) and 
transient bright dots (Tian et al. 2014). The scrutiny of this scenario requires a detailed analysis of the dynamics of the penumbral magnetic field in the chromosphere and of its coupling to the photosphere. A possible observational setup for this purpose would be similar to that presented in the current article, but either in sit-and-stare mode or with repetitive small raster scans (about $3^{\prime \prime}-4$ " wide) parallel to penumbral filaments. These would be accompanied by images in $\mathrm{Ca}$ II H or $\mathrm{K}$ and Interface Region Imaging Spectrograph (IRIS, De Pontieu et al. 2014) data.

Acknowledgements. The 1.5-m GREGOR solar telescope was built by a German consortium under the leadership of the Kiepenheuer-Institut für Sonnenphysik in Freiburg with the Leibniz-Institut für Astrophysik Potsdam, the Institut für Astrophysik Göttingen, and the Max-Planck-Institut für Sonnensystemforschung in Göttingen as partners, and with contributions by the Instituto de Astrofísica de Canarias and the Astronomical Institute of the Academy of Sciences of the Czech Republic. J.J. acknowledges support from the CHROMOBS project funded by the Kunt and Alice Wallenberg Foundation. This work was partly supported by the BK21 plus program through the National Research Foundation (NRF) funded by the Ministry of Education of Korea. This study is supported by the European Commissions FP7 Capacities Programme under the Grant Agreement number 312495 . The GRIS instrument was developed thanks to the support by the Spanish Ministry of Economy and Competitiveness through the project AYA2010-18 029 (Solar Magnetism and Astrophysical Spectropolarimetry).

\section{References}

Andretta, V., \& Jones, H. P. 1997, ApJ, 489, 375

Avrett, E. H., Fontenla, J. M., \& Loeser, R. 1994, in Infrared Solar Physics, eds.

D. M. Rabin, J. T. Jefferies, \& C. Lindsey, IAU Symp., 154, 35

Bard, S., \& Carlsson, M. 2008, ApJ, 682, 1376

Berkefeld, T., Soltau, D., Schmidt, D., \& von der Lühe, O. 2010, Appl. Opt., 49, G155

Berkefeld , T., Schmidt, D., Soltau, D., von der Lühe, O., \& Heidecke, F. 2012, Astron. Nachr., 333, 863

Borrero, J. M., \& Ichimoto, K. 2011, Liv. Rev. Sol. Phys., 8, 4

Borrero, J. M., Lites, B. W., \& Solanki, S. K. 2008, A\&A, 481, L13

Borrero, J. M., Asensio Ramos, A., Collados, M., et al. 2016, A\&A, 596, A2 (GREGOR SI)

Centeno, R., Trujillo Bueno, J., Uitenbroek, H., \& Collados, M. 2008, ApJ, 677, 742

Collados, M., Lagg, A., Díaz Garcí A, J. J., et al. 2007, in The Physics of Chromospheric Plasmas, eds. P. Heinzel, I. Dorotovič, \& R. J. Rutten, ASP Conf. Ser., 368, 611

Collados, M., López, R., Páez, E., et al. 2012, Astron. Nachr., 333, 872

Cuperman, S., Li, J., \& Semel, M. 1992, A\&A, 265, 296

De Pontieu, B., Title, A. M., Lemen, J. R., et al. 2014, Sol. Phys., 289, 2733

Esteban Pozuelo, S., Bellot Rubio, L. R., \& de la Cruz Rodríguez, J. 2015, ApJ, 803, 93

Franz, M., \& Schlichenmaier, R. 2013, A\&A, 550, A97

Franz, M., Collados, M., Bethge, C., et al. 2016, A\&A, 596, A4 (GREGOR SI)

Frutiger, C., Solanki, S. K., Fligge, M., \& Bruls, J. H. M. J. 2000, A\&A, 358, 1109
Joshi, J. 2014, Ph.D. Thesis, Technische Universität Braunschweig

Joshi, J., Pietarila, A., Hirzberger, J., et al. 2011, ApJ, 734, L18

Joshi, J., Lagg, A., Hirzberger, J., Solanki, S. K., \& Tiwari, S. K. 2016, A\&A, submitted

Jurčák, J., \& Katsukawa, Y. 2008, A\&A, 488, L33

Katsukawa, Y., Berger, T. E., Ichimoto, K., et al. 2007, Science, 318, 1594

Kuckein, C., Martínez Pillet, V., \& Centeno, R. 2012, A\&A, 539, A131

Lagg, A., Woch, J., Krupp, N., \& Solanki, S. K. 2004, A\&A, 414, 1109

Lagg, A., Ishikawa, R., Merenda, L., et al. 2009, in The Second Hinode Science Meeting: Beyond Discovery-Toward Understanding, eds. B. Lites, M. Cheung, T. Magara, J. Mariska, \& K. Reeves, ASP Conf. Ser., 415, 327

Langhans, K., Scharmer, G. B., Kiselman, D., Löfdahl, M. G., \& Berger, T. E. 2005, A\&A, 436, 1087

Leenaarts, J., Golding, T., Carlsson, M., Libbrecht, T., \& Joshi, J. 2016, A\&A, submitted

Lites, B. W., Elmore, D. F., Seagraves, P., \& Skumanich, A. P. 1993, ApJ, 418, 928

Livingston, W., \& Wallace, L. 1991, An atlas of the solar spectrum in the infrared from 1850 to $9000 \mathrm{~cm}^{-1}$ (1.1 to 5.4 micrometer), NSO Technical Report, National Solar Observatory

Magara, T. 2010, ApJ, 715, L40

Martínez Pillet, V. 2000, A\&A, 361, 734

Reardon, K., Tritschler, A., \& Katsukawa, Y. 2013, ApJ, 779, 143

Rempel, M., \& Schlichenmaier, R. 2011, Liv. Rev. Sol. Phys., 8, 3

Rüedi, I., Solanki, S. K., \& Livingston, W. C. 1995, A\&A, 293, 252

Ruiz Cobo, B., \& Asensio Ramos, A. 2013, A\&A, 549, L4

Sakurai, T., Makita, M., \& Shibasaki, K. 1985, in Theoretical Problems in High Resolution Solar Physics, ed. H. U. Schmidt, 313

Schad, T. A., Penn, M. J., Lin, H., \& Tritschler, A. 2015, Sol. Phys., 290, 1607

Scharmer, G. B., \& Henriques, V. M. J. 2012, A\&A, 540, A19

Scharmer, G. B., Henriques, V. M. J., Kiselman, D., \& de la Cruz Rodríguez, J. 2011, Science, 333, 316

Scharmer, G. B., de la Cruz Rodriguez, J., Sütterlin, P., \& Henriques, V. M. J. 2013, A\&A, 553, A63

Schmidt, W., Hofmann, A., Balthasar, H., Tarbell, T. D., \& Frank, Z. A. 1992, A\&A, 264, L27

Schmidt, W., von der Lühe, O., Volkmer, R., et al. 2012, Astron. Nachr., 333, 796

Solanki, S. K. 1987, Ph.D. Thesis, 8309, ETH, Zürich

Solanki, S. K. 2003, A\&ARv, 11, 153

Solanki, S. K., \& Montavon, C. A. P. 1993, A\&A, 275, 283

Tian, H., Kleint, L., Peter, H., et al. 2014, ApJ, 790, L29

Title, A. M., Frank, Z. A., Shine, R. A., et al. 1993, ApJ, 403, 780

Tiwari, S. K., van Noort, M., Lagg, A., \& Solanki, S. K. 2013, A\&A, 557, A25

Tiwari, S. K., van Noort, M., Solanki, S. K., \& Lagg, A. 2015, A\&A, 583, A119

Tiwari, S. K., Moore, R. L., Winebarger, A. R., \& Alpert, S. E. 2016, ApJ, 816, 92

van Noort, M., Lagg, A., Tiwari, S. K., \& Solanki, S. K. 2013, A\&A, 557, A24

Vissers, G. J. M., Rouppe van der Voort, L. H. M., \& Carlsson, M. 2015, ApJ, 811, L33

Wallace, L., Hinkle, K., \& Livingston, W. C. 1993, An atlas of the photospheric spectrum from 8900 to $13600 \mathrm{~cm}^{-1}$ (7350 to $11230 \AA$ ), NSO Technical Report 93-001 (National Solar Observatory)

Wilkinson, L. K., Emslie, G. A., \& Gary, G. A. 1989, Sol. Phys., 119, 77 